This paper was retracted by the publisher as it has numerous unfixable errors. In particular, the main result of the paper, Corollary 3.10, is unfortunately false. 
TAIWANESE JOURNAL OF MATHEMATICS

Vol. 24, No. 2, pp. 283 300 April 2020

DOI: $10.11650 / \mathrm{tjm} / 190703$

\title{
A Note on Number Knots and the Splitting of the Hilbert Class Field
}

\author{
Yih-Jeng Yu
}

\begin{abstract}
Several number knots are defined including the five knots introduced by W. Jehne. The question of the splitting of the group extension of the Hilbert class field can be read off in terms of the triviality of these knots.
\end{abstract}

\section{Introduction}

Let $K$ be a number field. We embed $K^{\times}$into the idele groys $\lambda$ and this gives rise to the exact sequence

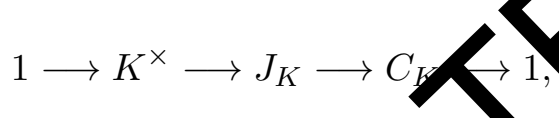

where $C_{K}$ is the idele class group of $K$. The ker of the nonical map $J_{K} \rightarrow I_{K}$ of the idele group onto the group of fractional ideals i the $y$ hit idele group $U_{K}$, giving rise to another exact sequence
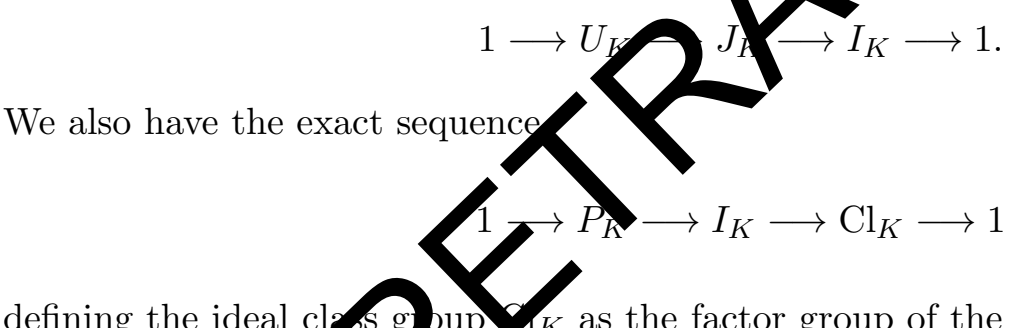

defining the ideal clo $\mathrm{g}$ bup ${ }_{K}$ as the factor group of the group $I_{K}$ modulo the group $P_{K}$ of principal idea All these three exact sequences fit into a commutative diagram, called the fundamental square,

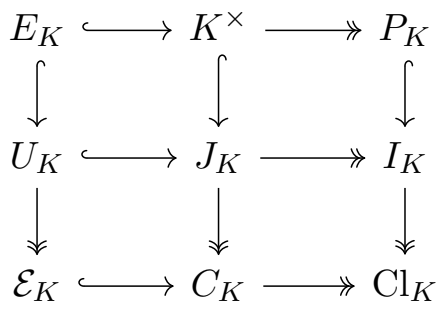

where $E_{K}$ is the global unit group and $\mathcal{E}_{K}$ is the idele unit class group.

Received August 23, 2018; Accepted July 3, 2019.

Communicated by Chia-Fu Yu.

2010 Mathematics Subject Classification. Primary: 12F10; Secondary: 11R32, 11R34.

Key words and phrases. number knots, Hilbert class field, abelian genus, central genus. 
For a Galois extension $K / k$ of number fields with Galois group $G=\operatorname{Gal}(K / k)$ and relative norm $N=N_{K / k}$, Jehne, in a seminal paper [12], applied the snake lemma to the following commutative diagram of certain abelian groups attached to number fields

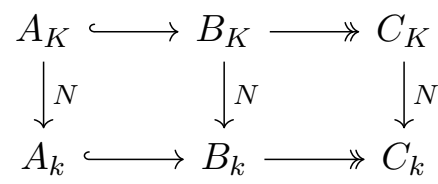

and obtained the exact sequence

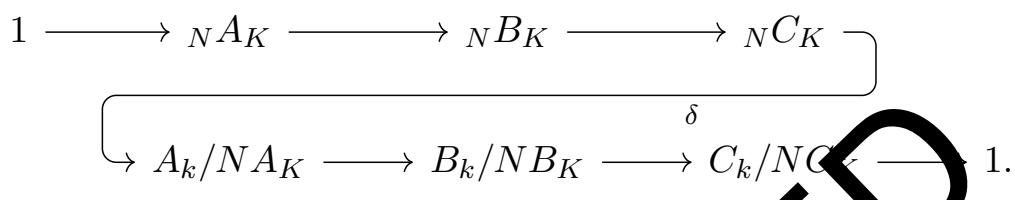

The image of the connecting homomorphism $\delta$ is $A_{k} \cap N B, \angle A A_{K}=[A, B]$, and he calls $[A, B]$ the knot associated to the exact sequence

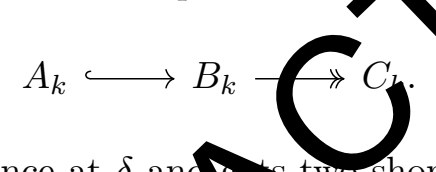

If one splits up the exact sequence at $\delta$ and go two short exact sequences involving the knot $[A, B]$ :

and

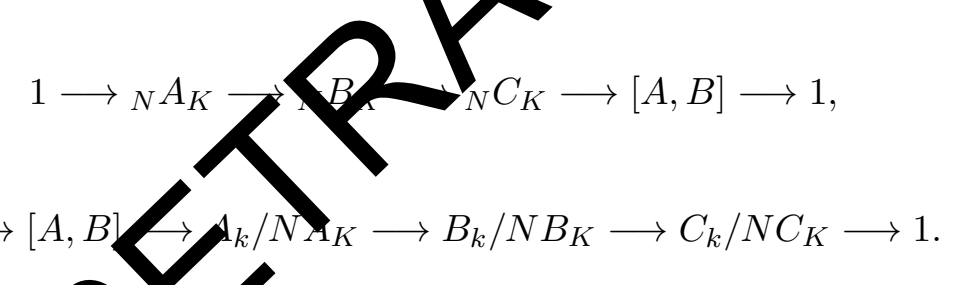

The exact sequences the fur mental square thus give rise to six knots (cf. 12, p. 220]):

$$
\begin{aligned}
{\left[U_{K}, J_{K}\right] } & =1 & & \\
\omega_{K / k} & :=\left[E_{K}, K^{\times}\right]=E_{k} \cap N K^{\times} / N E_{K} & & \text { first unit knot, } \\
\omega_{K / k}^{\prime} & :=\left[E_{K}, U_{K}\right]=E_{k} \cap N U_{K} / N E_{K} & & \text { second unit knot, } \\
\nu_{K / k} & :=\left[K^{\times}, I_{K}\right]=k^{\times} \cap N J_{K} / N K^{\times} & & \text {Scholz's number knot, } \\
\delta_{K / k} & :=\left[P_{K}, I_{K}\right]=P_{k} \cap N I_{K} / N P_{K} & & \text { divisor knot, } \\
\gamma_{K / k} & :=\left[\mathcal{E}_{K}, C_{K}\right]=\mathcal{E}_{k} \cap N C_{K} / N \mathcal{E}_{K} & & \text { idele class knot. }
\end{aligned}
$$

The vanishing of the knot $\left[U_{K}, J_{K}\right]$ of the idele units in the ideles reduces to the following statement:

$$
U_{\wp} \cap N_{K_{\ell} / k_{\wp}} K_{\ell}^{\times}=N_{K_{\ell} / k_{\wp}} U_{\ell}
$$

for $\ell / \wp$ in $K / k$. If $\wp$ is a finite place, for $\alpha=\pi^{n} \cdot u\left(u \in U_{\wp}, \pi\right.$ a prime element for $\ell$ ), the condition $N_{K_{\ell} / k_{\wp}}(\alpha)=\pi_{0}^{f n} \eta_{0} \cdot N_{K_{\ell} / k_{\wp}}(u) \in U_{\wp}\left(\eta_{0} \in U_{\wp}, \pi_{0}\right.$ a prime element for $\left.\wp\right)$ 
induces that $n=0$; so $\alpha \in U_{\ell}$. If $\wp$ is archimedian, we may assume that $\wp$ is real and $\ell$ is complex, and so both sides are the group of positive real numbers.

By the functorial properties of number knots, Jehne also proved the following fundamental knot sequence

$$
\omega_{K / k} \longrightarrow \omega_{K / k}^{\prime} \longrightarrow \nu_{K / k} \longrightarrow \delta_{K / k} \longrightarrow \gamma_{K / k}
$$

which extends the Scholz's knot sequence

$$
\omega_{K / k}^{0} \longleftrightarrow \nu_{K / k} \longrightarrow \delta_{K / k}^{0},
$$

where $\omega_{K / k}^{0}:=\omega_{K / k}^{\prime} / \omega_{K / k}$ and $\delta_{K / k}^{0}:=\operatorname{Im}\left(\nu_{K / k} \rightarrow \delta_{K / k}\right)$ are the Scholz's unit knot and Scholz's divisor knot, respectively.

Recall that an extension of number fields $K / k$ satisfies the He No $n$ Principle if any element of $k^{\times}$that is a norm everywhere locally is a global $\mathrm{m}$ fron The Hasse Norm Principle holds for the extension $K / k$ if and only if Scb $1 \mathrm{z}$ anot $\nu_{K / k}$ is trivial. Hasse [7] has shown that $\nu_{K / k}=1$ for cyclic extension $k$, and Scholz introduced knots in order to study the validity of Hasse Norm $\mathrm{P}$ nciple in Mon-cyclic cases. It is known that the Hasse Norm Principle holds for $K / k$ in ach 9 the following cases:

(1) there is a prime $\wp$ of $k$ such that $G_{\varphi}=C($ ar $[4, \S 11.4])$;

(2) the least common multiple of ocounds $[K: k]$ (cf. [12]).

Moreover, Scholz's number knot $\nu_{\Lambda_{k}}$ cam also be related to the Schur multiplier $H^{2}(G$, $\mathbb{Q} / \mathbb{Z}$ ) of the Galois group

The knots defined and an of certain subfields in the Hilber lar $H_{K}$ of $K\left[12\right.$, Theorem 3]: The abelian genus field $H_{K}^{\prime}$ of $K$ over $k$ is the maxim unramified abelian extension of $K$ that is of the form $E K$ where $E$ is an abelian extension of $k$. The central class field $H_{K}^{*}$ of $K$ over $k$ is the maximal unramified extension of $K$ such that $H_{K}^{*}$ is Galois over $k$ and $\operatorname{Gal}\left(H_{K}^{*} / K\right)$ is contained in the center of $\operatorname{Gal}\left(H_{K}^{*} / k\right)$. Obviously, we have $H_{K}^{\prime} \subseteq H_{K}^{*} \subseteq H_{K}$. For original definition, see [12, p. 228]. Then, we have

$$
\delta_{K / k}^{0} \simeq \operatorname{Gal}\left(H_{K}^{*} / H_{K}^{\prime}\right), \quad \delta_{K / k} \simeq \operatorname{Gal}\left(H_{K}^{*} / H_{k} K\right) \quad \text { and } \quad \gamma_{K / k} \simeq \operatorname{Gal}\left(H_{K}^{\prime} / H_{k} K\right) .
$$

The field tower of Galois extensions $H_{K} / K / k$ defines a group extension with abelian kernel and factor set $\alpha$ :

$$
\alpha: 1 \longrightarrow \operatorname{Gal}\left(H_{K} / K\right) \longrightarrow \operatorname{Gal}\left(H_{K} / k\right) \longrightarrow G \longrightarrow 1 \text {. }
$$

By class field theory, the abelian kernel is isomorphic to the ideal class group $\mathrm{Cl}_{K}$ of $K$ via the Artin map: $\operatorname{Gal}\left(H_{K} / K\right) \simeq \mathrm{Cl}_{K}$. By the theorem of Weil and Shafarevich, the 
two-cohomology class $[\alpha]$ is the image of the fundamental class $u=u_{K / k}$ of $K / k$ under the natural map

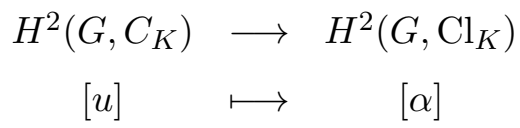

induced by the map $C_{K} \rightarrow \mathrm{Cl}_{K}$. It is a well-known fact that $H^{2}\left(G, C_{K}\right)$ is isomorphic to the cyclic group generated by $u$.

The question of the splitting of the group extension (1.3), or equivalently, of the triviality of the map (1.4) has been studied by several authors. Herz 10 has originally believed that the group extension $(1.3)$ always splits for $k=\mathbb{Q}$. Wyman $[20]$ showed that this is true provided that the Galois group $G$ is cyclic; however, h gan a counter-example in the abelian case. In the same paper, Wyman also gave asu cient condition for the splitting of (1.3) which involves a certain cohomological kn G d, h, 6, Theorem 2], gave a simplified proof of Wyman's result in the cyclic cas Cor all and Rosen [5], provided another proof of Wyman-Gold's results and gave necessa conditions and examples for the splitting [5, Proposition 7] which involves cholz divisor knot $\delta_{K / k}^{0}$. Bond found necessary and sufficient conditions in som case of belian unramified extension $K / k$ (see [2, Theorem 3.1, Proposition 3.7, and Cr 1rasy 3.9]).

In this note, we introduce furth co omd ogical knots $\nu_{n}(K / k)$ for $n=-1,0,2$ and $\tau_{K / k}$ in Section 2. We also relate hem with the above number knots of Scholz and Jehne. Moreover, in Section 3, we yish point out that the splitting of the group extension (1.3) can be read off in te ns triviality of certain cohomological knots. To this end, we define a global inertia s up an we then prove our main theorem (Theorem 3.8) which states that if the ar hrondition of Wyman holds, the global inertia group equals to the Galois group itse Our main theorem yields, for abelian Galois group, a sufficient condition for the splitting of the group extension $(1.3)$ is $H_{k} \cap K=k$ (cf. Corollary 3.10). The above corollary of our main theorem generalizes a result of Wyman and Gold [6, 20], which is the preceding statement when $G$ is cyclic.

In the final section, we introduce the group $J$ which can be identified with a subgroup of $\operatorname{Ker}(j)$, where $j: \mathrm{Cl}_{k} \rightarrow \mathrm{Cl}_{K}$ is the extension map of ideals. In a recent paper of Bond [3], he studies the capitulation problem for arbitrary abelian extensions using cohomological methods to describe $\operatorname{Ker}(j)$ and $\operatorname{Coker}(j)$. He investigates the intersection $J$ of the image of the norm map from $\mathrm{Cl}_{K}$ to $\mathrm{Cl}_{k}$ with $\operatorname{Ker}(j)$, and shows that if $J$ is trivial then $\operatorname{Ker}(j)$ is isomorphic to the Galois group of $L$ over $k$ where $L$ is the intersection of $K$ with the Hilbert class field of $k$. We also provide examples of splitting and of non-splitting of the group extension 1.3 which can be read off in terms of the triviality of certain cohomological knots (cf. Theorem 4.5). Bond's necessary and sufficient conditions mentioned above 
are direct consequence of a cohomological characterization of finite nilpotent groups, the Scholz's knot sequence, and our knots $\nu_{0}$ and $v_{-1}$.

\section{Number knots of a Galois extension}

Let $K / k$ be a Galois extension of number fields with Galois group $G=\operatorname{Gal}(K / k)$. Consider the following fundamental square of $G$-modules:

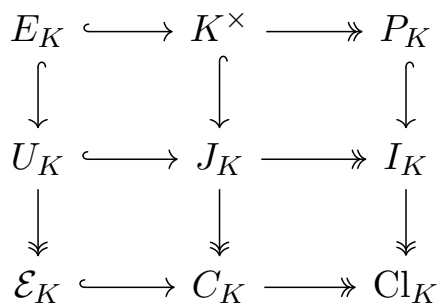

Applying Tate cohomology to the second and third loye quepces of the above fundamental square, we obtain, for $n \in \mathbb{Z}$, the followin diagram with exact rows

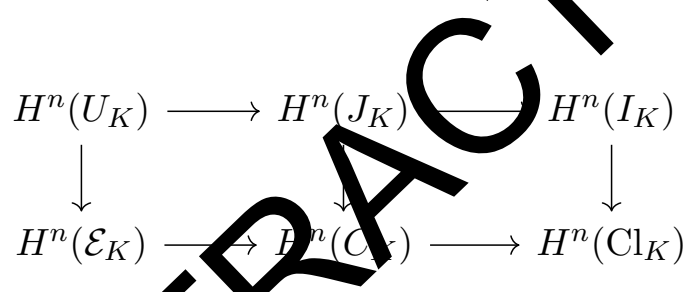

where $H^{n}(\cdot)$ is the usual abbre the Tate cohomology group $H^{n}(G, \cdot)$. For any $n \in \mathbb{Z}$, we define the followig cohon logical knot

$$
22^{n}=\operatorname{Coker}\left(H^{n}\left(\mathcal{E}_{K}\right) \rightarrow H^{n}\left(C_{K}\right)\right) .
$$

For places $\ell / \wp$ of $/ k$, let $T_{\ell} \leq G_{\ell}$ be the inertia subgroup and the decomposition group of $K / k$ at $\ell / \wp$, respectively. Let $\pi G=H_{2}(G, \mathbb{Z})$ be the fundamental group of $G$. Since $\mathbb{Q} / \mathbb{Z}$ is an injective abelian group, $\operatorname{Ext}\left(H_{1}(G, \mathbb{Z}), \mathbb{Q} / \mathbb{Z}\right)$ is trivial and hence, by the universal coefficient theorem, $H^{2}(G, \mathbb{Q} / \mathbb{Z}) \stackrel{\simeq}{\longrightarrow} \operatorname{Hom}\left(H_{2}(G, \mathbb{Z}), \mathbb{Q} / \mathbb{Z}\right)$. That is, $\pi G$ is the Pontryagin dual of the Schur multiplier $H^{2}(G, \mathbb{Q} / \mathbb{Z})$ of $G$. If, in addition, the group $G$ is cyclic, then $\pi G={ }_{N} \mathbb{Z} / I_{G} \mathbb{Z}$ is trivial where $I_{G}$ is the augmentation ideal.

We now recall a well-known result of Tate [18, p. 198], one part of which has been discovered by Scholz [14, Satz 3]. See also [12, p. 221].

Theorem 2.1 (Scholz-Tate). The Scholz's number knot $\nu_{K / k}$ is the cokernel of the map $\coprod_{\wp, \ell / \wp} \pi G_{\ell} \rightarrow \pi G$ of the fundamental groups:

$$
\nu_{K / k} \simeq \operatorname{Coker}\left(\coprod_{\wp, \ell / \wp} \pi G_{\ell} \rightarrow \pi G\right)
$$


Therefore, the number knot $\nu_{K / k}$ is a quotient of the Pontryagin dual of Schur multiplier $H^{2}(G, \mathbb{Q} / \mathbb{Z})$. We define the cohomological knot $\tau_{K / k}$ to be the cokernel of the map $\coprod_{\wp, \ell / \wp} \pi T_{\ell} \rightarrow \pi G$ :

$$
\tau_{K / k}:=\operatorname{Coker}\left(\coprod_{\wp, \ell / \wp} \pi T_{\ell} \rightarrow \pi G\right) .
$$

To see the canonical map $\tau_{K / k} \rightarrow \nu_{K / k}$ is surjective, we can apply the kernel-cokernel exact sequence to the pair of maps

$$
\coprod_{\wp, \ell / \wp} \pi T_{\ell} \stackrel{f}{\longrightarrow} \coprod_{\wp, \ell / \wp} \pi G_{\ell} \stackrel{g}{\longrightarrow} \pi G
$$

which yields an exact sequence

$$
\cdots \longrightarrow \operatorname{Ker}(g) \longrightarrow \operatorname{Coker}(f) \longrightarrow \tau_{K / k}
$$

The following results are important consequences globa a local class field theory (cf. [13, 15, 18), and they are fundamental to the moin re lts of this paper:

Proposition 2.2. Let $K$ be a finite abelian ext (sion) ver $k$ with Galois group $G$.

(1) $H^{1}\left(K^{\times}\right)=1$ (Hilbert's Theorem 90).

(2) For any $n \in \mathbb{Z}$, the map - where $u \in H^{2}\left(C_{K}\right)$ is the fundamental class, is an orph. In particular, we have $H^{0}\left(C_{K}\right) \simeq H^{-2}(\mathbb{Z}) \simeq$ G (see [18, p. 197]).

(3) For any $n \in \mathbb{Z}$, $A^{n}\left(J_{K}\right) \simeq \coprod_{\wp} H^{n}\left(G_{\ell}, K_{\wp}^{\times}\right) \simeq \coprod_{\wp} H^{n-2}\left(G_{\ell}, \mathbb{Z}\right)$, where $G_{\ell}$ denotes th $d$ sition group for an $\ell \mid \wp$ and $\wp$ runs over all places of k. In particular, we have $H^{0}\left(J_{K}\right) \simeq \coprod_{\wp} H^{-2}\left(G_{\ell}, \mathbb{Z}\right) \simeq \coprod_{\wp} G_{\ell}$ and $H^{1}\left(J_{K}\right)=1$ (see [18, p. 139 and p. 177]).

(4) $U_{K}^{G}=U_{k}$ and $H^{0}\left(U_{K}\right) \simeq \coprod_{\wp} T_{\ell}$, where $T_{\ell}$ denotes the inertia group for an $\ell \mid \wp$.

(5) $H^{-1}\left(I_{K}\right)=H^{1}\left(I_{K}\right)=1$.

By (1.4) and the bottom line of (2.1), the splitting of 1.3$)$ is equivalent to the triviality of the cohomological knot $\nu_{2}(K / k)$ :

Theorem 2.3. The group extension (1.3) splits if and only if

$$
\nu_{2}(K / k)=1 \text {. }
$$

Corollary 2.4 (Gold [6]). Suppose that $G$ is cyclic. The splitting of $(1.3$ is equivalent to $\nu_{0}(K / k)=1$. 
Proof. Since $G$ is cyclic, by the two-periodicity of the cohomology of a cyclic group, we thus have

$$
\begin{aligned}
\nu_{2} & :=\operatorname{Coker}\left(H^{2}\left(\mathcal{E}_{K}\right) \rightarrow H^{2}\left(C_{K}\right)\right) \\
& \simeq \operatorname{Coker}\left(H^{0}\left(\mathcal{E}_{K}\right) \rightarrow H^{0}\left(C_{K}\right)\right)=: \nu_{0},
\end{aligned}
$$

and the result follows from Theorem 2.3 .

To get necessary conditions for the splitting, we make use of the theorem of WeilShafarevich in class field theory. If $\nu_{2}(K / k)=1$, there exists $[\eta] \in H^{2}\left(\mathcal{E}_{K}\right)$ such that $[\eta] \mapsto[u]$. Now the functorial property of cup-products (cf. [4, Chapter 4, $\S]$ ) with $[u]$, $[\eta]$ and $[\alpha]$ induces, for $n=0$ and -1 , the following commutatiy diag am

where the middle vertical map $H^{n-2}(\mathbb{Z}) \rightarrow$ an isomorphism by Proposition 2.2 .

From the above commutative diagr an noting that

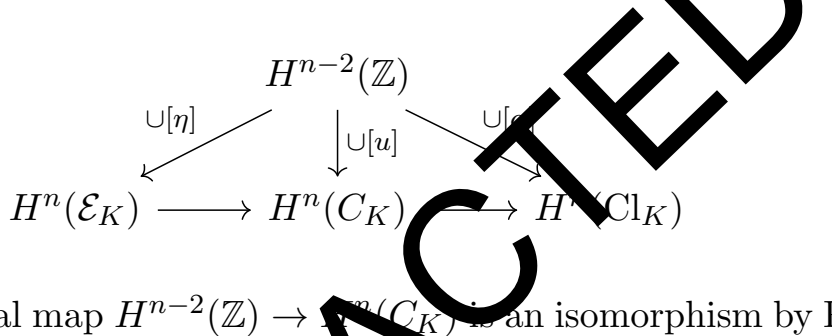


To describe $\nu_{-1}(K / k)$ and $\tau_{K / k}$, we consider our exact and commutative diagram (2.1) for $n=-1$ taking into account the fact that $H^{-1}\left(I_{K}\right)=1$ (see Proposition 2.2 (5)):

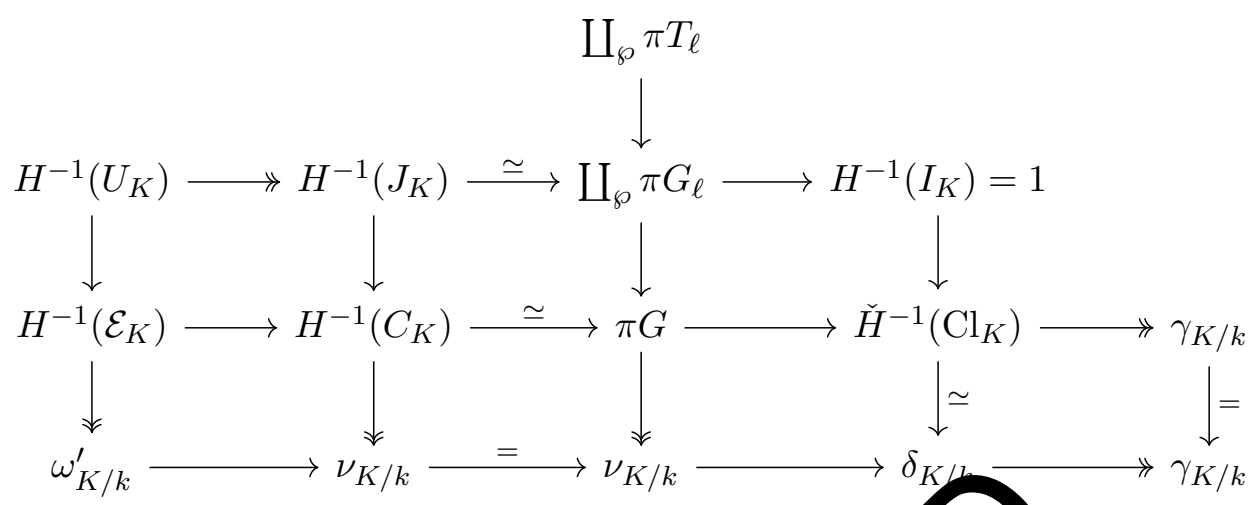

where $\check{H}^{-1}\left(\mathrm{Cl}_{K}\right)={ }_{N} \mathrm{Cl}_{K} /{ }_{N} I_{K}$. If we let $\delta_{K / k}^{0}$ be the Scholy div or hot, we can easily prove the following:

Lemma 2.8. (1) Let $H_{K}^{*}$ and $H_{K}^{\prime}$ be the central genus dhe abelian genus of $H_{K} / K / k$, respectively. We have $\nu_{-1}(K / k) \simeq \delta_{K / k}^{0} \simeq \operatorname{aal}\left(H_{K}^{*} / H_{K}\right)$.

(2) The following assertions are equivale

(a) the map $H^{-1}\left(U_{K}\right) \rightarrow H^{-1} C_{K}$ is urjective.

(b) $\nu_{K / k}=1$.

(3) If $\tau_{K / k}=1$, then $\nu_{K}$

Proof. (1) The diagrar 2 .2 act and commutative by 12 , Theorem 1]. Since $\check{H}^{-1}\left(\mathrm{Cl}_{K}\right)$ $\simeq \delta_{K / k}$, we can view ${ }_{k}\left(\nu_{K / k} \rightarrow \delta_{K / k}\right)$ as a subgroup of $\check{H}^{-1}\left(\mathrm{Cl}_{K}\right)$ and so we have

$$
\begin{aligned}
\delta_{K / k}^{0} & :=\operatorname{Im}\left(\nu_{K / k} \rightarrow \delta_{K / k}\right) \simeq \operatorname{Ker}\left(\check{H}^{-1}\left(\mathrm{Cl}_{K}\right) \rightarrow \gamma_{K / k}\right) \\
& =\operatorname{Im}\left(\pi G \rightarrow \check{H}^{-1}\left(\mathrm{Cl}_{K}\right)\right) \simeq \operatorname{Coker}\left(H^{-1}\left(\mathcal{E}_{K}\right) \rightarrow H^{-1}\left(C_{K}\right)\right)=: \nu_{-1}(K / k) .
\end{aligned}
$$

(2) Since $H^{-1}\left(I_{K}\right)=1$ by Proposition $2.2(5)$, the map $H^{-1}\left(U_{K}\right) \rightarrow H^{-1}\left(J_{K}\right)$ is surjective. The exact and commutative diagram (2.2) reveals that $\nu_{K / k}=1$ is equivalent to the map $H^{-1}\left(U_{K}\right) \rightarrow H^{-1}\left(C_{K}\right)$ is surjective.

(3) The result is obvious, since $\tau_{K / k} \rightarrow \nu_{K / k}$ is surjective.

Remark 2.9. The referee has remarked that, in Lemma 2.8(2), the Scholz's number knot $\nu_{K / k}$ is actually isomorphic to $\operatorname{Coker}\left(H^{-1}\left(U_{K}\right) \rightarrow H^{-1}\left(C_{K}\right)\right)$. This follows by applying the kernel-cokernel exact sequence to the pair of maps

$$
H^{-1}\left(U_{K}\right) \stackrel{f}{\longrightarrow} H^{-1}\left(J_{K}\right) \stackrel{g}{\longrightarrow} H^{-1}\left(C_{K}\right)
$$


which yields an exact sequence

$$
\cdots \longrightarrow 1=\operatorname{Coker}(f) \longrightarrow \operatorname{Coker}(g \circ f) \longrightarrow \operatorname{Coker}(g)=\nu_{K / k} \longrightarrow 1
$$

Lemmas 2.5, 2.8(1), and the Scholz's knot sequence (1.2) yield

Corollary 2.10. If 1.3 splits, then $\delta_{K / k}^{0}$ is trivial, or equivalently, the map $\omega_{K / k}^{\prime} \rightarrow \nu_{K / k}$ is surjective by the exactness of (1.1). In other words, if (1.3) splits, then $k^{\times} \cap N J_{K}=$ $\left(E_{k} \cap N U_{K}\right) N K^{\times}\left(\subseteq J_{k}\right)$.

\section{Wyman's sufficient condition for the splitting}

We let $e_{0}$ be the least common multiple of all the ramification in aces $K / k$, finite and infinite. Since each ramification index $e_{\wp}$ divides $[K: k]$, we divides $[K: k]$ as well.

A rather strong sufficient arithmetic condition giv by following:

\section{Theorem 3.1 (Wyman [20]). For an arbitrary $a$, if}

then the group extension (1.3) splits

The original proof of Wym a base class field theory; later, Gold [6, Theorem 1], and Cornell and Rosen [5. ropositia 5] gave simplified proofs for Wyman's result.

The splitting of 1,3 ans triviality of $\nu_{2}(K / k)=\operatorname{Coker}\left(H^{2}\left(\mathcal{E}_{K}\right) \rightarrow H^{2}\left(C_{K}\right)\right)$ which can be read fro $\mathrm{h}$ thullowing commutative diagram:

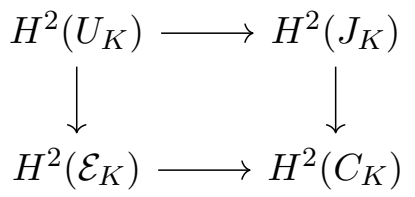

In fact, the splitting is ensured by the surjectivity of the following map $g \circ f$ :

$$
H^{2}\left(U_{K}\right) \stackrel{f}{\longrightarrow} H^{2}\left(J_{K}\right) \stackrel{g}{\longrightarrow} H^{2}\left(C_{K}\right)
$$

Hence, to prove Wyman's result is to show that the arithmetic condition (3.1) implies the surjectivity of $g \circ f$.

Remark 3.2. The map $g$ is part of the exact sequence

$$
1 \longrightarrow H^{2}\left(K^{\times}\right) \longrightarrow H^{2}\left(J_{K}\right) \stackrel{g}{\longrightarrow} H^{2}\left(C_{K}\right) \stackrel{\delta}{\longrightarrow} H^{3}\left(K^{\times}\right) \longrightarrow 1,
$$


which is induced from the following exact sequence of $G$-modules

$$
1 \longrightarrow K^{\times} \longrightarrow J_{K} \longrightarrow C_{K} \longrightarrow 1
$$

with $H^{1}\left(C_{K}\right)=1$ by global class field theory (cf. [18, Theorem $9.1(2)$, p. 180]). Thus, this map $g$ is surjective provided that $H^{3}\left(K^{\times}\right)=1$ which is the case for a cyclic extension $K / k$, by the two-periodicity of the cohomology of a cyclic group and by Hilbert's Theorem 90 . Moreover, $g$ is also surjective if

$$
[K: k]=\operatorname{lcm}\left\{\left|G_{\ell}\right|: \ell / \wp, \wp \text { a place of } k\right\},
$$

where $G_{\ell}$ is the decomposition group of $K / k$ at $\ell / \wp$. The proof is based on the fact about the cohomology of idele groups that $H^{2}\left(G, J_{K}\right) \simeq \prod_{\wp} H^{2}\left(G_{\ell}, K_{\ell}^{\times}\right)$and via invariant maps. See [20, §4].

We now give a proof of Wyman's result:

Theorem 3.3. The arithmetic condition 3.1 implies th jectivty of $g \circ f$. In other words, the arithmetic condition (3.1) implies the split of $\mathbf{\gamma}$ ert class field.

Proof. Since the global invariant map inv : $H^{2}\left(C \nrightarrow \frac{1}{[K: k]} / \mathbb{Z}\right.$ is an isomorphism (cf. 18 , bottom of p. 196]), it suffices to show that it is p sible o take, for each place $\wp$, the local factor system $u_{\wp}(G) \in H^{2}\left(G, U_{\wp}\right)$ with inva rar inv $\left(u_{\wp}(G)\right)=\frac{\lambda_{\wp}}{e_{\wp}}+\mathbb{Z}$.

First, we have $\left.H^{2}\left(U_{K}\right)=\prod_{\wp} H^{2}(\cap)\right)$, there

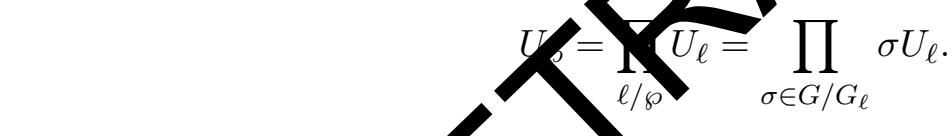

Hence, for $\ell / \wp$ we have $t$ is morphism $H^{2}\left(G, U_{\ell}\right) \simeq H^{2}\left(G_{\ell}, U_{\ell}\right)$ by Shapiro's Lemma. By Serre 16 , p. 167 Cor Criction map Cor $H^{2}\left(T_{\ell}, U_{\ell}\right) \rightarrow H^{2}\left(G_{\ell}, U_{\ell}\right)$ is injective. Finally, by W $H^{2}\left(T_{\ell}, K_{\ell}^{\times}\right)$is an isom cphism and the group $H^{2}\left(T_{\ell}, U_{\ell}\right)$ has order $e_{\wp}$. Altogether, we have

$$
H^{2}\left(T_{\ell}, K_{\ell}^{\times}\right) \stackrel{\simeq}{\longrightarrow} H^{2}\left(T_{\ell}, U_{\ell}\right) \stackrel{\text { Cor }}{\longrightarrow} H^{2}\left(G_{\ell}, U_{\ell}\right) \stackrel{\simeq}{\longrightarrow} H^{2}\left(G, U_{\ell}\right) .
$$

Therefore, $\operatorname{inv}\left(H^{2}\left(G, U_{\wp}\right)\right)$ contains $\frac{1}{e_{\wp}}$ for all ramified $\wp$.

Now the fundamental class $u=u_{K / k} \in H^{2}\left(C_{K}\right)$ has invariant

$$
\operatorname{inv}(u)=\frac{1}{[K: k]}+\mathbb{Z} \in \mathbb{Q} / \mathbb{Z} .
$$

The arithmetic condition 3.1 implies the solvability in $\lambda_{\wp} \in \mathbb{Z}$ of the congruence equation

$$
\sum_{\wp} \frac{\lambda_{\wp}}{e_{\wp}} \equiv \frac{1}{[K: k]} \quad \bmod \mathbb{Z} .
$$

From this, we show the surjectivity of $g \circ f$ by taking for each place $\wp$ the local factor system $u_{\wp}(G) \in H^{2}\left(G, U_{\wp}\right)$ with invariant $\operatorname{inv}\left(u_{\wp}(G)\right)=\frac{\lambda_{\wp}}{e_{\wp}}+\mathbb{Z}$. 
Using arguments analogous as in the proof of Bemerkung (4) of [8, p. 41], one shows that the exponent of $\tau_{K / k}$ divides $[K: k] / e_{0}$. In particular, the arithmetic condition (3.1) implies the triviality of $\tau_{K / k}$ hence that of Scholz's number knot $\nu_{K / k}$ by Lemma 2.8(3).

Corollary 3.4. If the arithmetic condition (3.1) holds, then $\tau_{K / k}=\nu_{K / k}=1$.

However, the splitting of 1.3 does not necessarily imply $\tau_{K / k}=\nu_{K / k}=1$, as we shall see later.

Remark 3.5. If $K / k$ is a unramified cyclic extension of degree $n$, Hilbert's Theorem 94 says that $|\operatorname{Ker}(j)|$ is divisible by $n$, where $j: \mathrm{Cl}_{k} \rightarrow \mathrm{Cl}_{K}$ is the extension map of ideals. This result was extended to all unramified abelian extensions by Suzuki [17]. Moreover, if $L=H_{k} \cap K$, the maximal unramified abelian extension of $k$ in is a cyclic extension of $k$, then $|\operatorname{Ker}(j)|$ is divisible by $n / e_{0}$.

In the rest of this section, we will show how far is the th cohomological knot $\tau_{K / k}$ related to the splitting problem. To this end, will a duce the global inertia group and prove a group theoretic result.

Definition 3.6. For a Galois extension $K / k$ o numb $/$ fields with Galois group $G$, we define the global inertia group $T$ to be the g ory orenerated by all inertia groups $T_{\ell}$ of $\ell / \wp$ for $K / k$, i.e.,

We let $\widetilde{T}$ be the normal subgroup $G$ generated by $T$, that is, $\widetilde{T}=T \cdot[T, G]$.
Remark 3.7. (1) Obvigusly $T \leq \widetilde{T} \leq G$.

(2) Let $L=H_{k} \quad K$ It is well-known that $\operatorname{Gal}(K / L)$ is the global inertia group $T$. Moreover, if $\operatorname{Gal}\left(K / L\right.$, is cyclic, we have $[K: L]=e_{0}$. See [20, p. 147].

(3) Suppose that $K / k$ has a totally ramified prime $\wp$. Let $T_{\ell}$ be the inertia subgroup in the extension $H_{K} / k$ of some prime $\ell$ lying over $\wp$. Note that $T_{\ell} \cap \operatorname{Gal}\left(H_{K} / K\right)=1$ and $T_{\ell} \cdot \operatorname{Gal}\left(H_{K} / K\right) / \operatorname{Gal}\left(H_{K} / K\right)=G$. Hence, $T_{\ell}$ is a complement for $\operatorname{Gal}\left(H_{K} / K\right)$ in $\operatorname{Gal}\left(H_{K} / k\right)$ and the group extension (1.3) splits. Moreover, if $T$ is the inertia subgroup in $K / k$ of some ramified prime and $K^{T}$ is the fixed field of $T$, then the group extension

$$
1 \longrightarrow \operatorname{Gal}\left(H_{K} / K\right) \longrightarrow \operatorname{Gal}\left(H_{K} / K^{T}\right) \longrightarrow T \longrightarrow 1
$$

splits. Here, the above-mentioned group extension is the restriction of (1.3) to $T$. Thus, each restriction of (1.3) to an inertia subgroup of $G$ splits.

We now state our first main theorem as follows:

Theorem 3.8. (1) If $e_{0}=[K: k]$, we have $G=\widetilde{T}=T$. 
(2) For arbitrary Galois group $G$, the followings are equivalent:

(i) $G=\widetilde{T} \cdot G^{\prime}=T \cdot G^{\prime}$, where $G^{\prime}=[G, G]$ is the commutator subgroup of $G$.

(ii) $N \mathrm{Cl}_{K}=\mathrm{Cl}_{k}$.

(iii) $H_{k} \cap K=k$.

Proof. (1) The assumption says that $[K: k]=e_{0}=\prod_{p} p^{n_{p}}$, hence $p^{n_{p}}$ divides some $e_{\wp}$ which is the largest $p$-power dividing the latter. By the above, we have the Sylow subgroups $\operatorname{Syl}_{p}(G)=\operatorname{Syl}_{p}\left(T_{\ell}\right)$ for $\ell / \wp / p$; thus proves $(1)$ :

$$
G=\left\langle\bigcup_{p \mid[K: k]} \operatorname{Syl}_{p}(G)\right\rangle \leq\left\langle\bigcup_{p \mid[K: k]} \operatorname{Syl}_{p}\left(T_{\ell}\right)\right\rangle \leq T \leq \widetilde{T} .
$$

(2) The equivalence of (ii) and (iii) is well-known (see Le ma 2. B). By class field theory (cf. Proposition 2.2(2), (3), and (4)), we have comp cible orphisms inducing the following commutative diagram

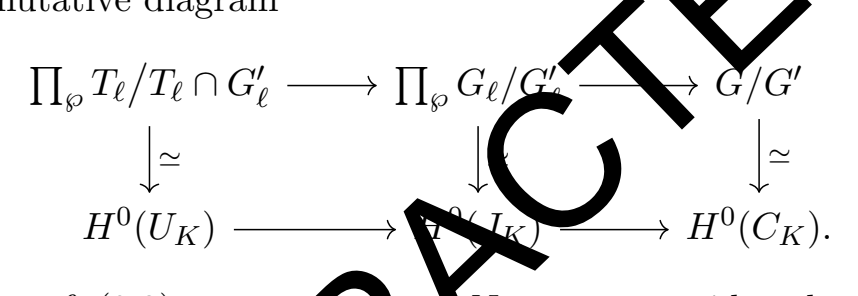

Note that the rows of 3.2 are $n g$ ex Now, we consider the two bottom exact sequences of the diagram $\left(1.6^{*}\right)$ [12. 221 :

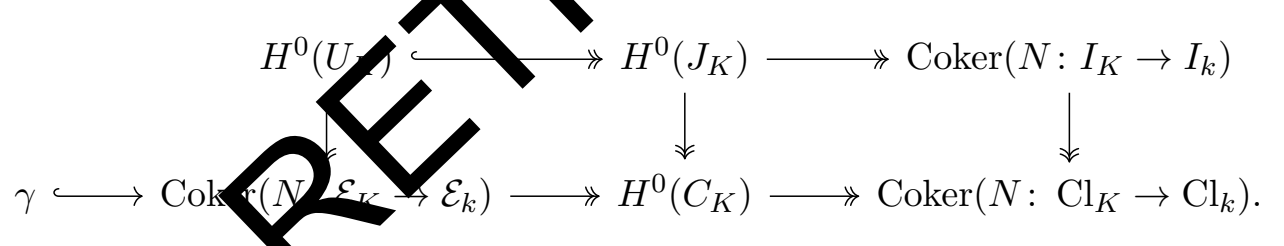

It follows from (3.2) tha he the condition (i) is equivalent to the surjectivity of $H^{0}\left(U_{K}\right) \rightarrow$ $H^{0}\left(J_{K}\right)$ by Second Isomorphism Theorem, which is equivalent to the triviality of Coker $(N$ : $\left.I_{K} \rightarrow I_{k}\right)$ hence of $\operatorname{Coker}\left(N: \mathrm{Cl}_{K} \rightarrow \mathrm{Cl}_{k}\right)$, i.e., the condition (ii) $N \mathrm{Cl}_{K}=\mathrm{Cl}_{k}$.

To apply our theorem to finite solvable groups, we need the following easy result from group theory.

Lemma 3.9. Let $G$ be a finite solvable group, and $H$ be a subgroup of $G$. If $G=\widetilde{H} \cdot G^{\prime}=$ $H \cdot G^{\prime}$, then we obtain $G=\widetilde{H}$.

Proof. First, we see that $\widetilde{H}=H \cdot[H, G]$. Since $G$ is solvable, the $t$-th commutator subgroup $G^{(t)}$ is trivial for some $t \in \mathbb{N}$. Now, we have $G^{\prime}=\left[\widetilde{H} \cdot G^{\prime}, \widetilde{H} \cdot G^{\prime}\right] \leq \widetilde{H} \cdot G^{(2)}$. Hence, by induction, we have $G^{(i)} \leq \widetilde{H} \cdot G^{(i+1)}$; therefore, we obtain

$$
G^{(t-1)} \leq \widetilde{H}
$$


and

$$
G \leq \widetilde{H} \cdot G^{\prime} \leq \widetilde{H} \cdot G^{(2)} \leq \cdots \leq \widetilde{H}
$$

This proves the assertion.

For abelian group $G$, the two groups $T$ and $\widetilde{T}$ coincide. In this case, Theorem 3.8 and Lemma 3.9 say that the condition $H_{k} \cap K=k$ implies the equality of the Galois group $G$ of $K / k$ with its global inertia group: $G=T=\widetilde{T}$. For this reason, Wyman's sufficient condition (3.1) for the splitting boils down to $H_{k} \cap K=k$ which generalizes the cyclic case (cf. Corollary 2.7).

Corollary 3.10. If $G$ is abelian, a sufficient condition for the splitting of (1.3) is $H_{k} \cap K$ $=k$.

Remark 3.11. The equalities $G=T=\widetilde{T}$ does not necessarily $\mathrm{m}_{\mathrm{v}}$ the splitting of (1.3), as we shall see in the following. First, the equalities $G T T$ Might not imply the arithmetic condition (3.1): $[K: k]=e_{0}$. For example t $p$ d be two rational primes congruent to 1 modulo $4, K=\mathbb{Q}(\sqrt{p}, \sqrt{q})$, and $k=$ Since $\mathbb{Q}$ has no unramified extensions, we have $G=T$. There are two rami cationsubgroups, each of order 2 . Thus $e_{0}=2 \neq 4=[K: k]=|G|$.

Now, we come to our counter-example: et $\mathbb{Q}\left(\sqrt{p_{1}}, \sqrt{p_{2}}, \sqrt{p_{3}}, \sqrt{p_{4}}\right)$ where $p_{i}$ are rational primes congruent 1 modulo an $\mathrm{d}$ le $k=\mathbb{Q}$. For this extension, the hypothesis of Corollary 3.10 is fulfilled, in $\mathrm{f} C \mathrm{et}=\mathbb{Q}$. As before, we have $G=T$, but the group extension 1.3 does not split sincone can show that $\delta_{K / k}^{0} \neq 1$, contrary to Lemmas 2.5 and 2.6 .

Problem 3.12. Sin $\Omega=\widetilde{T}$ does not necessarily implies the arithmetic condition (3.1), the above manuron $G=T=\widetilde{T}$ might not sufficient for the splitting. What additional condition to condition $H_{k} \cap K=k$ will imply the splitting in the abelian case?

\section{Some examples}

Various examples of splitting and of non-splitting of the group extension (1.3) which appeared in the papers of Cornell and Rosen [5, Proposition 9], and Bond [2, Theorem 3.1, Propositions 3.7, 3.8, and Corollary 3.9] can be read off in terms of the triviality of certain number knots and by simple group theoretic arguments.

Example 4.1. Associated to the group extension (1.3) with factor set $\alpha$ and abelian kernel $A:=\mathrm{Cl}_{K}$ is the central group extension with factor set $\alpha^{*}$ :

$$
1 \longrightarrow A /[A, E] \longrightarrow E /[A, E] \longrightarrow G \longrightarrow 1
$$


where $E=\operatorname{Gal}\left(H_{K} / k\right), A /[A, E] \simeq \operatorname{Gal}\left(H_{K}^{*} / K\right)$ and $E /[A, E] \simeq \operatorname{Gal}\left(H_{K}^{*} / k\right)$. Note that (4.1) is the pushforward of $(1.3)$ along $E \rightarrow E /[A, E]$, so that the splitting of (1.3), i.e., $\alpha=1$, implies that of 4.1 .

Recall that a central extension is a (non-split) group extension where the base normal subgroup is contained in the center of the whole group. It is a central stem extension if, in addition, the base normal subgroup is contained in the derived subgroup of the whole group. Therefore, a central stem extension never splits. Examples of non-splitting of (4.1) now can be supplied. To illustrate this, by assuming $K=H_{K^{\prime}}$, we have $A \leq[E, E]$, and thus the extension (4.1) is a stem extension. So the example of non-splitting in Cornell and Rosen [5, Proposition 9] is still valid without any assumption on the class number of K.

To generalize Bond's examples, we first define a group

$$
J:=\operatorname{Coker}\left(H^{-1}\left(C_{K}\right) \rightarrow H^{-1}\right.
$$

Remark 4.2. We let $j: \mathrm{Cl}_{k} \rightarrow \mathrm{Cl}_{K}$ be the extension map f ideals. In [3, Bond shows:

(1) The group $J$ is a subgroup of $\operatorname{Ker}(j)$ co sisting of those ideal classes of $k$ that are in the image of the norm map fro $\mathrm{Cl}_{K} \mathrm{Cl}_{k}$ and that capitulate in $K$, i.e., $J=\operatorname{Ker}(j) \cap N \mathrm{Cl}_{K}$. Moreover $H=k$, we have $\operatorname{Ker}(j)=J$. See also Proposition 0.2 and Remark 2 of

(2) There exists an unramifiea ab lian extension $M$ of $k$ such that $\operatorname{Ker}(j) / J \simeq \operatorname{Gal}(L / M)$, where $L=H_{k} \cap K$ and $J \mid$ is divisible by $[M: k]$ by Suzuki's Theorem. Hence, if $J=1$, by $\operatorname{Remar} 2.5$ t is asy to see that $\operatorname{Ker}(j) \simeq \operatorname{Gal}(L / k)$, that is, $M=k$. See also [3, Theor n 2 b]

Recall that $H^{-1}\left(\mathrm{Cl}_{K}\right)={ }_{N} \mathrm{Cl}_{K} / I_{G} \mathrm{Cl}_{K}$. The group $J$ fits in into a part of the commutative diagram with exact rows of 2.2 (cf. [12, Theorem 1]):

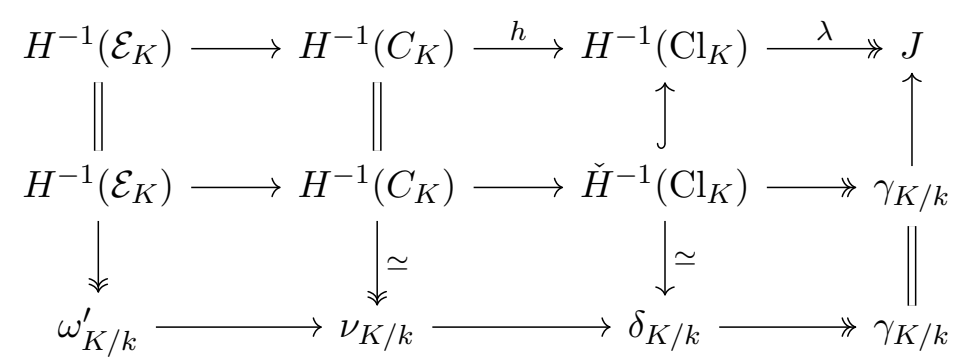

where $\check{H}^{-1}\left(\mathrm{Cl}_{K}\right)={ }_{N} \mathrm{Cl}_{K} /{ }_{N} I_{K} \simeq \delta_{K / k}$.

Remark 4.3. Let $\alpha$ be the class of the group extension 1.3. If the group extension (1.3) splits, then $\lambda$ is an isomorphism. 
Indeed, letting $u \in H^{2}\left(C_{K}\right)$ be the fundamental class, the map $H^{-3}(\mathbb{Z}) \rightarrow H^{-1}\left(C_{K}\right)$ defined by $x \mapsto u \cup x$ is an isomorphism by Proposition 2.2(2). We consider the following commutative diagram:

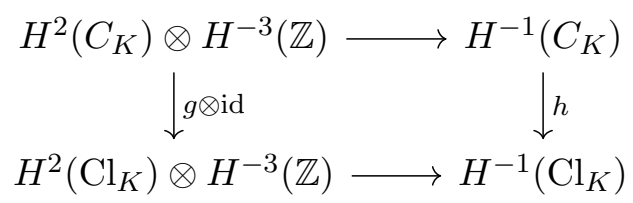

We know that $g(u)=\alpha$. Let $z \in H^{-1}\left(C_{K}\right)$. Then $z=u \cup x$ for some $x \in H^{-3}(\mathbb{Z})$, and so $h(z)=g(u) \cup x=\alpha \cup x$. Since the group extension (1.3) splits, we have $\alpha=1$; thus, we derive that $h(z)=1 \cup x$ and hence $\lambda$ is an isomorphism.

We need the following cohomological characterization of fip e nh otent groups (see [11, 19]):

Lemma 4.4. A finite group $G$ is nilpotent if and only whe $M$ is a finite $G$-module for which $H^{k}(G, M)=1$ for some $k$, then $H^{n}(G, M=$ for all $n \in \mathbb{Z}$.

Lemma 4.4 and the diagram (4.2) above lead us eas y to the following general results:

Theorem 4.5. Let $K / k$ be a finite Galois ete with the Scholz's number knot $\nu_{K / k}$. Suppose that $\nu_{K / k} \simeq H^{-1}\left(C_{K}\right) \simeq H, G$, L $)$, Pontryagin dual of the Schur multiplier of $G$. Then we have:

(1) The splitting of 1.3 amplies at $\nu_{-1}=1$ and $H^{-1}\left(\mathrm{Cl}_{K}\right) \simeq J$. In particular, we have $\omega_{K / k}^{0} \simeq \nu_{K / k}$.

(2) Suppose that is Imotent. If $\nu_{-1}=1$ and $J=1$, then (1.3) splits.

Proof. (1) Suppose that the group extension (1.3) splits. By Lemma 2.5, we have $\nu_{-1}=$ $1=\nu_{0}$; by Lemma $2.8(1)$, we see that $\delta^{0}=\nu_{-1}=1$. Reading off in the diagram 4.2, this is equivalent to saying that $\omega_{K / k}^{0} \simeq \nu_{K / k}$ and $\check{H}^{-1}\left(\mathrm{Cl}_{K}\right) \simeq \gamma$. Hence, $H^{-1}\left(\mathcal{E}_{K}\right) \rightarrow$ $H^{-1}\left(C_{K}\right) \simeq \nu_{K / k}$ is surjective, and so $H^{-1}\left(\mathrm{Cl}_{K}\right) \simeq J$.

(2) If $J=1$, we have $H^{-1}\left(C_{K}\right) \rightarrow H^{-1}\left(\mathrm{Cl}_{K}\right)$ is surjective. If $\nu_{-1}=1$, the Scholz's divisor knot $\delta^{0}=1$ by Lemma $2.8(1)$; thus, by Scholz's knot sequence (1.2), we see that $\omega_{K / k}^{0} \simeq \nu_{K / k}$ and hence $H^{-1}\left(\mathcal{E}_{K}\right) \rightarrow H^{-1}\left(C_{K}\right)$ is also surjective. Reading off in the diagram 4.2), these imply that $H^{-1}\left(\mathrm{Cl}_{K}\right)=1$. Since $G$ is nilpotent, we obtain that $H^{2}\left(\mathrm{Cl}_{K}\right)=1$; thus this map $H^{2}\left(C_{K}\right) \rightarrow H^{2}\left(\mathrm{Cl}_{K}\right)$ is a trivial map and this is equivalent to the splitting of 1.3 .

Remark 4.6. It should be noticed that if $J=1$, then every ideal of $k$ which capitulates in $K$ capitulates in $H_{k} \cap K$. Indeed, if $j_{L / k}: \mathrm{Cl}_{k} \rightarrow \mathrm{Cl}_{L}$ is the extension map of ideals, 
where $L=H_{k} \cap K$, then it is easy to see that $\operatorname{Ker}\left(j_{L / k}\right) \subseteq \operatorname{Ker}(j)$. By Lemma 2.6, the norm map $N_{K / L}: \mathrm{Cl}_{K} \rightarrow \mathrm{Cl}_{L}$ is surjective. Therefore, we have $N \mathrm{Cl}_{K}=N_{L / k} \mathrm{Cl}_{L}$ and $\operatorname{Ker}\left(j_{L / k}\right) \cap N_{L / k} \mathrm{Cl}_{L} \subseteq \operatorname{Ker}(j) \cap N \mathrm{Cl}_{K}=J$. By Remark 4.2, we have $\operatorname{Ker}\left(j_{L / k}\right)=\operatorname{Ker}(j)$.

Theorem 4.5 covers the following Bond's examples:

Example 4.7. Let $k$ be an imaginary quadratic field and $K / k$ be an abelian unramified extension of odd degree. In the unramified case, all decomposition groups are cyclic, and $N U_{K}=U_{k}$. Thus, the Scholz's number knot $\nu_{K / k} \simeq H^{-1}\left(C_{K}\right) \simeq H^{-3}(\mathbb{Z})$, and the Scholz's unit knot $\omega_{K / k}^{0}:=E_{k} \cap N U_{K} / E_{k} \cap N K^{\times}=E_{k} / E_{k} \cap N K^{\times}=1$, since $E_{k}=\{ \pm 1\} \leq N K^{\times}$, by assumption on $k$ and the odd degree of $K / k$. Moreover, in the abelian unramified case, $J$ can be identified with $\operatorname{Ker}(j) \cap N \mathrm{Cl}_{K}$ (see [1, Theorem 1]). By Theorem 4.5, necessary and sufficient conditions for the splitting of 1.3 in this case th $G$ be cyclic and $J=1$. See also Theorem 3.1 of 2 .

Example 4.8. Let $k$ be an imaginary quadratic field $h$ d $h$ the unramified abelian extension of degree $2^{t}, t \geq 1$. Suppose that -1 is not ne norm of a unit of $K$. Since the Scholz's unit knot $\omega^{0}$ has order 1 or 2 , depe ding on whether or not $-1 \in N K^{\times}$, the splitting of 1.3 is equivalent to $J=1$, and whe her $t$ e Schur multiplier of $G$ has order 1 or 2 . The latter is known to be depending n ther $G$ is cyclic or $G \simeq \mathbb{Z}_{2^{t-1}} \times \mathbb{Z}_{2}$. So we have two cases for the splitting:

(1) If $G \simeq \mathbb{Z}_{2^{t-1}} \times \mathbb{Z}_{2}$, then split is equivalent to $J=1$ and -1 is not a global norm from $K$.

(2) If $G$ is cyclic, the spli ing is equivalent to $J=1$.

See also Propositions 7, 3.8, and Corollary 3.9 of [2].

\section{Acknowledgments}

We thank the anonymous referee for a careful reading of the original manuscript and many helpful suggestions.

\section{References}

[1] R. J. Bond, Some results on the capitulation problem, J. Number Theory 13 (1981), no. 2, 246-254.

[2] _ On the splitting of the Hilbert class field, J. Number Theory 42 (1992), no. 3, 349-360. 
[3] - Capitulation in abelian extensions of number fields, Acta Arith. 179 (2017), no. 3, 201-232.

[4] J. W. S. Cassels and A. Fröhlich, Algebraic Number Theory, Academic Press, London New York, 1967.

[5] G. Cornell and M. Rosen, A note on the splitting of the Hilbert class field, J. Number Theory 28 (1988), no. 2, 152-158.

[6] R. Gold, Hilbert class fields and split extensions, Illinois J. Math. 21 (1977), no. 1, 66-69.

[7] H. Hasse, Die Normenresttheorie relativ-Abelscher Zahlkörper als Klassenkörpertheorie im Kleinen, J. Reine Angew. Math. 162 (1930), 145-154.

[8] F.-P. Heider, Zur Theorie der zahlentheoretischen Kr ten Dissutation, Köln, 1978.

[9] Z_ Zahlentheoretische Knoten unendlicher iterungen, Arch. Math. (Basel) 37 (1981), no. 4, 341-352.

[10] C. S. Herz, Construction of class field 91, Lecture Notes in Mathematics 21, Spor-Verlag, New York, 1966.

[11] K. Hoechsmann, P. Roquetto a A Mossenhaus, A cohomological characterization of finite nilpotent groups, Ma (Basel) 19 (1968), 225-244.

[12] W. Jehne, On knots agebraic number theory, J. Reine Angew. Math. 311/312 (1979), 215-254

[13] J. Neukirch, Alg braic Number Theory, Grundlehren der Mathematischen Wissenschaften 322, Translated from the 1992 German original and with a note by Norbert Schappacher, With a foreword by G. Harder, Springer-Verlag, Berlin, 1999.

[14] A. Scholz, Totale Normenreste, die keine Normen sind, als Erzeuger nichtabelscher Körpererweiterungen II, J. Reine Angew. Math. 182 (1940), 217-234.

[15] J.-P. Serre, Local class field theory, in: Algebraic Number Theory (Proc. Instructional Conf., Brighton, 1965), 128-161, Thompson, Washington, D.C., 1967.

[16] Local Fields, Graduate Texts in Mathematics 67, Springer-Verlag, New York, 1979.

[17] H. Suzuki, A generalization of Hilbert's theorem 94, Nagoya Math. J. 121 (1991), 161-169. 
[18] J. T. Tate, Global class field theory, in: Algebraic Number Theory (Proc. Instructional Conf., Brighton, 1965), 162-203, Thompson, Washington, D.C., 1967.

[19] W. J. Wong, A cohomological characterization of finite nilpotent groups, Proc. Amer. Math. Soc. 19 (1968), 689-691.

[20] B. F. Wyman, Hilbert class fields and group extensions, Scripta Math. 29 (1973), 141-149.

Yih-Jeng Yu

Department of Mathematical Sciences, National Chengchi University, 11605 Taipei, Taiwan

E-mail address: yjyu@nccu.edu.tw 